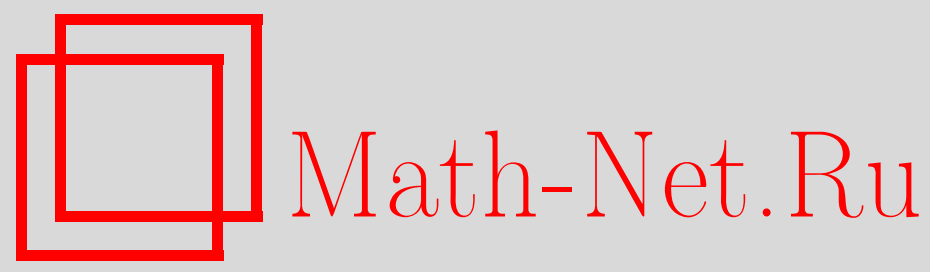

В. Л. Верещагин, Асимптотическое поведение сингулярных в нуле решений уравнения синус-Гордон, Матем. заметки, 2000, том 67, выпуск 3, 329-342

DOI: https://doi.org/10.4213/mzm846

Использование Общероссийского математического портала Math-Net.Ru подразумевает, что вы прочитали и согласны с пользовательским соглашением http://www.mathnet.ru/rus/agreement

Параметры загрузки:

IP : 52.6 .47 .48

26 апреля 2023 г., 16:09:09

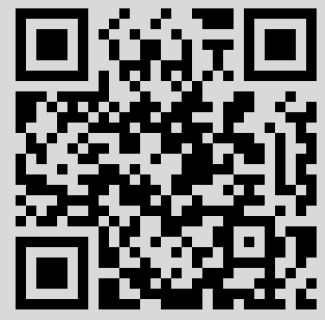




\section{АСИМПТОТИЧЕСКОЕ ПОВЕДЕНИЕ СИНГУЛЯРНЫХ В НУЛЕ РЕШЕНИЙ УРАВНЕНИЯ СИНУС-ГОРДОН}

\section{В. Л. Верещагин}

Поставлен вопрос об асимптотическом исследовании радиально симметричных решений уравнения синус-Гордон, сводимых к третьему трансценденту Пенлеве. Изучаются решения, имеющие сингулярность в начале координат. Получено асимптотическое разложение такого решения в области конечных значений независимой переменной, так что главным членом является модулированная эллиптическая функция. Выписаны соответствующие уравнение модуляций и сдвиг фазы.

Библиография: 9 названий.

1. Введение. Широко известное эллиптическое уравнение синус-Гордон

$$
u_{t t}+u_{y y}+\sin u+f=0
$$

имеет класс сингулярных в нуле решений с особенностями вида

$$
u \approx \alpha \log \frac{1}{x}+\beta+o(1), \quad x^{2}=y^{2}+t^{2} \rightarrow 0
$$

( $\alpha, \beta$ - параметры), которые выделяются среди множества других своим особьм значением для приложений. Так, эти решения служат для моделирования точечных дефектов в физике конденсированного состояния. Их видная роль в скалярных теориях поля обусловлена тем, что в отличие от гладких решений (1.1) сингулярные имеют конечную энергию

$$
E=\int\left[\frac{1}{2} u_{t}^{2}+\frac{1}{2} u_{y}^{2}-\cos u+\cos u_{0}-f\left(u-u_{0}\right)\right] d t d y
$$

Таким образом, решения с особенностями вида (1.2) могут быть использованы для описания связанньх состояний в теории поля. Более подробное обсуждение прикладного значения решений (1.2), а также соответствующая библиография приведены в статье [1]. Эта работа положила начало асимптотическому исс ледованию упомянутых сингулярных решений (1.2) с точки зрения метода изомонодромных деформаций. Авторы изучали случай радиально симметричного уравнения (1.1):

$$
u_{x x}+\frac{1}{x} u_{x}+\sin u+f=0
$$

Работа выполнена при финансовой поддержке Российского фонда фундаментальных исследований, грант № 97-01-00049. 
которое при $f=0$ является специальным видом уравнения Пенлеве третьего типа $\left(P_{3}\right)$. Оказалось, что решения уравнения (1.3), имеющие в начале координат особенность вида (1.2), стремятся к асимптотическому пределу на бесконечности (см. [2]):

$$
u(x)=2 \pi k+\gamma \sqrt{\frac{\pi}{2 x}} e^{-x}\left[1+O\left(x^{-1}\right)\right], \quad x \rightarrow \infty
$$

где $K$ - целое число. Асимптотические формулы для третьего трансцендента Пенлеве при больших значениях $x$ были получены с помошью метода усреднения в работе [3]. Специальньй интерес вызьвает поведение этих решений в конечной области - на не очень больших расстояниях они образуют волновой пакет типа ряда солитонов. Проведенные авторами работы [1] численные эксперименты описывают упомянутые осциллящии с помощью следующих графов:

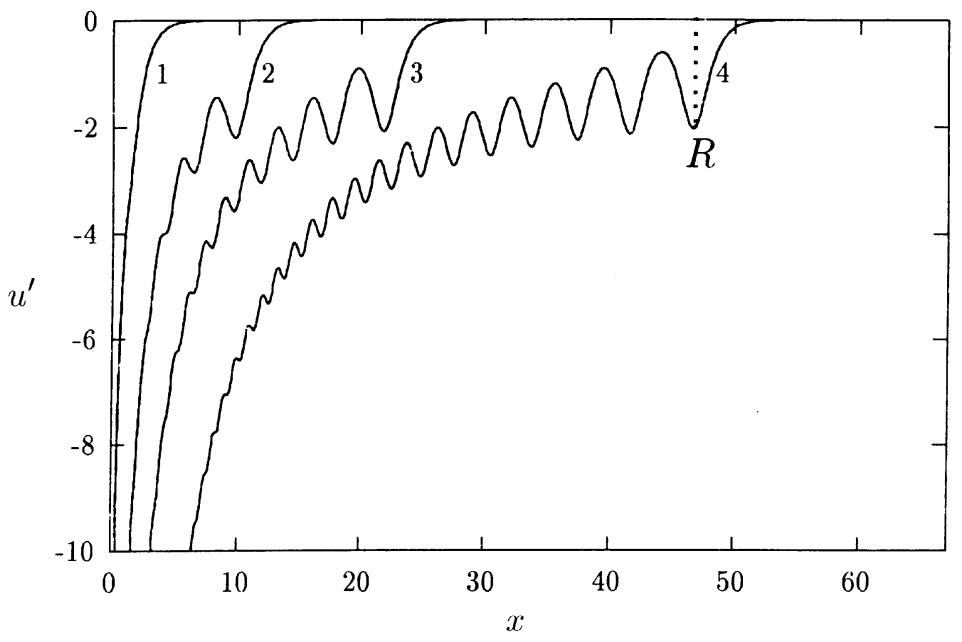

Рис. 1

Здесь разные кривые соответствуют разньм значениям параметра $\alpha$ в формуле (1.2). Величина $R$ обозначает координату последнего солитонного пика. Заметно, что с ростом значения параметра $\alpha$ решение формирует характерную "уиземову" зону осцилляций, причем линейная зависимость правой границы $R$ зоны от параметра $\alpha$ иллюстрируется на рис. 2 .

Перечисленньми наблюдениями была определена главная цель данной работы - с помощью формализма усреднения описать асимптотическое (при $\alpha \rightarrow \infty$ ) поведение сингулярных решений уравнения (1.3) для $f=0$ в области конечных значений переменной $x$. Основным результатом является представление главного члена этой асимптотики в виде модулированной эллиптической функции, определяюшей упомянутые вьше осциллящии. Метод квантования однозонного потенциала, описанный в статье [4], задает модуляции параметров эллиптической функции. Далее, в п. 3, ставится задача вычисления асимптотического распределения нулей функции $\sin u$ с помощью метода изомонодромных деформаций (см. [5]).В следующем пункте применение метода ВКБ [6] 


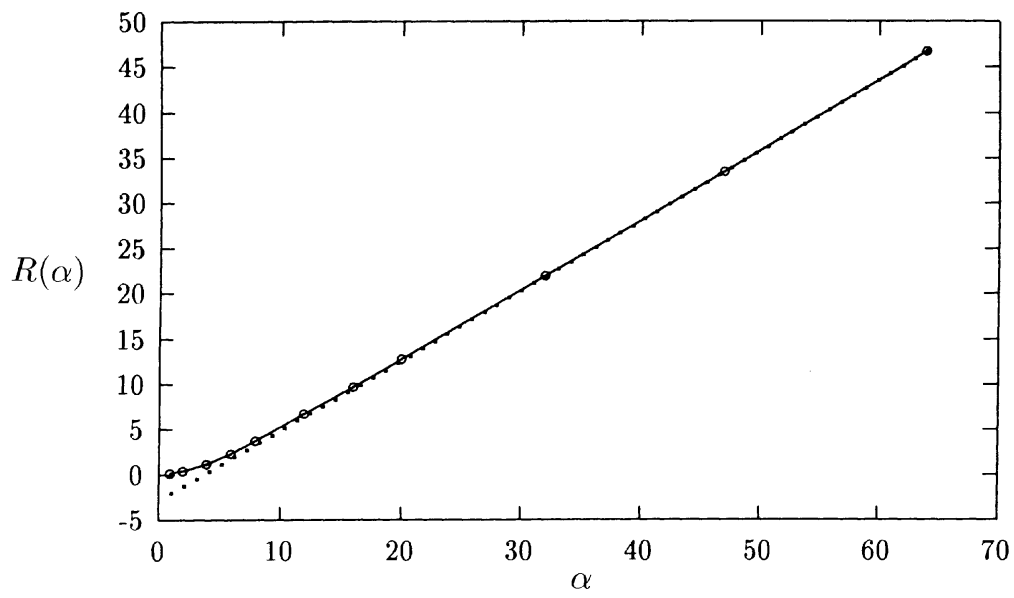

Рис. 2

в окрестности точек поворота позволяет вычислить множители Стокса - первые интегралы третьего уравнения Пенлеве (1.3). Пятьй пункт посвящен вычислению нулей функции $\sin u$ и, как следствие, фазы упомянутой выше эллиптической функции.

2. Метод усреднения для уравнений Пенлеве. Шесть уравнений Пенлеве известны как интегрируемые нелинейные обыкновенные дифференциальные уравнения. Вероятно, наиболее наглядным образом этот факт иллюстрируется наличием матричного коммутативного представления

$$
D_{\lambda} L_{j}-D_{x} A_{j}+\left[L_{j}, A_{j}\right]=0, \quad j=1,2, \ldots, 6,
$$

где $L_{j}=L_{j}\left(u, u^{\prime}, x, \lambda\right), A_{j}=A_{j}\left(u, u^{\prime}, x, \lambda\right), D_{\lambda}=\partial / \partial \lambda-2 \times 2$-матрицы, рационально зависящие от спектрального параметра $\lambda$, так что равенство (2.1) эквивалентно $j$-му уравнению Пенлеве. Матрицы $L_{j}, A_{j}$ приведены в статье [7]. Введем теперь величину $X=X(x)$ и заменим ею все переменные $x$, явно входящие в матрицы $L_{j}, A_{j}$ : $L_{j}=L_{j}\left(u, u^{\prime}, X, \lambda\right), A_{j}=A_{j}\left(u, u^{\prime}, X, \lambda\right)$. Тогда верны следующие предложения, иллюстрируемые ниже на конкретном примере третьего уравнения Пенлеве.

ПРЕДЛОЖЕНИЕ 2.1. Пусть $\varepsilon-$ некоторое положстельное число. Тогда выполнение равенства

$$
\varepsilon D_{\lambda} L_{j}-D_{x} A_{j}+\left[L_{j}, A_{j}\right]=0, \quad j=1,2, \ldots, 6
$$

әквивалентно системе

$$
X^{\prime}=\varepsilon, \quad u^{\prime \prime}-P_{j}\left(u, u^{\prime}, X\right)=0,
$$

где $u^{\prime \prime}=P_{j}\left(u, u^{\prime}, x\right)-j$-е уравнение Пенлеве. 
ПРЕДЛОЖЕНИЕ 2.2. Решение уравнения (2.2) при $\varepsilon=0, X=$ const может быть представлено в виде

$$
u_{0}(x)=f_{j}(\tau+\varphi ; \bar{a}),
$$

әде $\tau=x U, f_{j}$ - эллиптическая функиия (см. [8]), $U=U(X), \varphi=\varphi(X) ; \bar{a}(X)-$ параметры, определяющие функиию $f_{j}$.

Предположим теперь, что $\varepsilon$ - малая положительная величина. Будем искать решение системы (2.3) в виде формального ряда по $\varepsilon$ :

$$
u(x)=\varepsilon^{-1} u_{-1}(X)+u_{0}(x)+\varepsilon u_{1}(x)+O\left(\varepsilon^{2}\right),
$$

так что параметры $\bar{a}(X)$, определяющие функцию $u_{0}=f_{j}$, подчиняются некоторому обыкновенному дифференциальному уравнению, называемому системой Уизема или уравнением модулящий.

Главньй член ряда (2.5) (величина порядка $\varepsilon^{-1}$ ) может быть легко определен для $P_{3}$ путем подстановки (2.5) в само уравнение Пенлеве:

$$
u_{-1}=\log X
$$

Таким образом, нужно найти следуюший член разложения (2.4) в виде

$$
u_{0}(\tau, X)=f_{j}\left(\frac{S(X)}{\varepsilon}+\varphi(X) ; \bar{a}(X)\right), \quad D_{X} S=U
$$

Лемма 2.1. Система Уизема для уравнений Пенлеве имеет следующий вид:

$$
D_{X} \operatorname{det} A_{j}=\overline{a_{22} D_{\lambda} l_{11}}+\overline{a_{11} D_{\lambda} l_{22}}-\overline{a_{12} D_{\lambda} l_{21}}-\overline{a_{21} D_{\lambda} l_{12}}, \quad j=1,2, \ldots 6
$$

где $A_{j}=\left(a_{m n}\right), L_{j}=\left(l_{m n}\right), m, n=1,2 ;$ черта сверху означает усреднение по периоду әллиптической функиии $f_{j}(2.4)$.

ДокАЗАТЕЛЬСтво. Легко видеть, что из уравнения (2.2) при $\varepsilon=0$ вытекает независимость спектральных характеристик матрицы $A_{j}$ от переменной $x$. В частности, выполняется условие $\partial_{x} \operatorname{det} A_{j}=0$. Введение формальной переменной $X$ индуцирует следующую замену правила дифференцирования функций вида (2.4): $\partial_{x} \mapsto U \partial_{\tau}+\varepsilon \partial_{X}$. Для малого параметра $\varepsilon>0$ уравнение (2.2) обеспечивает выполнение равенства $a_{n, m}^{\prime}=\varepsilon \partial_{\lambda} l_{n, m}+\left[L_{j}, A_{j}\right]_{n, m}$. Подставляем последнее условие в тождество

$$
\partial_{x} \operatorname{det} A_{j}=a_{11}^{\prime} a_{22}+a_{11} a_{22}^{\prime}-a_{12}^{\prime} a_{21}-a_{12} a_{21}^{\prime}
$$

меняем закон дифференцирования и получаем по модулю величин порядка $O\left(\varepsilon^{2}\right)$ следующее:

$$
\left(U \partial_{\tau}+\varepsilon \partial_{X}\right) \operatorname{det} A_{j}=\varepsilon\left(a_{22} \partial_{\lambda} l_{11}+a_{11} \partial_{\lambda} l_{22}-a_{12} \partial_{\lambda} l_{21}-a_{21} \partial_{\lambda} l_{12}\right) .
$$

Далее усредняем, т.е. интегрируем по периоду $\tau$. Процедура усреднения уничтожает величины, являющиеся полными производными по $\tau$, из чего вытекает требуемое. 
ПРЕДЛОЖЕнИЕ 2.3. Существует только один коэффициент полинома по $\lambda$, ивя динамика по $X$ не тривиальна (исключением является шестое уравнение Пенлеве). Обозначим его $F_{j}$. Таким образом, система Уизема может быть записана в виде одного обыкновенного дифференциального уравнения первого порядка на величину $F_{j}$.

ПРЕДЛоЖенИЕ 2.4. Система Уизема (2.8) обеспечивает выполнение следующего формального условия:

$$
\left(\varepsilon^{-1} u_{-1}+u_{0}\right)^{\prime \prime}-P_{j}\left(\varepsilon^{-1} u_{-1}+u_{0},\left(\varepsilon^{-1} u_{-1}+u_{0}\right)^{\prime}, x\right)=O(\varepsilon) .
$$

В конкретном случае третьего уравнения Пенлеве $(j=3)$

$$
u^{\prime \prime}+\frac{1}{x} u^{\prime}+\sin u=0
$$

приведенные выше формуль имеют следующий вид:

$$
A=i\left(\frac{\cos u}{\lambda^{2}}-\frac{x^{2}}{16}\right) \sigma_{3}-\frac{i x u^{\prime}}{4 \lambda} \sigma_{1}+\frac{i \sin u}{\lambda^{2}} \sigma_{2} ; \quad L=-\frac{i x \lambda}{8} \sigma_{3}-\frac{i u^{\prime}}{2} \sigma_{1},
$$

әде

$$
\sigma_{1}=\left(\begin{array}{cc}
0 & 1 \\
1 & 0
\end{array}\right), \quad \sigma_{2}=\left(\begin{array}{cc}
0 & i \\
-i & 0
\end{array}\right), \quad \sigma_{3}=\left(\begin{array}{cc}
1 & 0 \\
0 & 1
\end{array}\right) .
$$

Система (2.2) выглядит так:

$$
X^{\prime}=\varepsilon, \quad X\left(u^{\prime \prime}+\sin u\right)+X^{\prime} u^{\prime}=0,
$$

прямое вычисление определителя матрицы $A_{j}$ дает равенство

$$
\lambda^{4} \operatorname{det} A=\frac{\lambda^{4} X^{4}}{256}-\frac{\lambda^{2} X^{2}}{8} F+1,
$$

где параметр $F$ появляется в качестве константы интегрирования второго уравнения системы (2.12) при $\varepsilon=0$ :

$$
F=\cos u-\frac{1}{2}\left(u^{\prime}\right)^{2} .
$$

Подставляя конкретные матричные әлементы в формулу (2.8), получаем

$$
D_{X} \operatorname{det} A=\frac{X^{3}}{64}-\frac{X \overline{\cos u}}{4 \lambda^{2}} .
$$

С другой стороны, дифференцируем по Х равенство (2.13) и выводим явный вид уравнения модулячий (ср. с [3])

$$
D_{X} F=-\frac{2}{X}(\overline{\cos u}-F)=\frac{1}{X}\left[\frac{17}{16}\left(F+\sqrt{F^{2}-1}\right) \frac{E}{K}-2 F\right],
$$

где $E, K$ - полные әллиптические интеграль:

$$
K=\int_{0}^{1} \frac{d z}{\sqrt{\left(1-z^{2}\right)\left(1-k z^{2}\right)}}, \quad E=\int_{0}^{1} \sqrt{\frac{1-k z^{2}}{1-z^{2}}} d z, \quad k=\frac{2 \sqrt{F^{2}-1}}{F+\sqrt{F^{2}-1}} .
$$




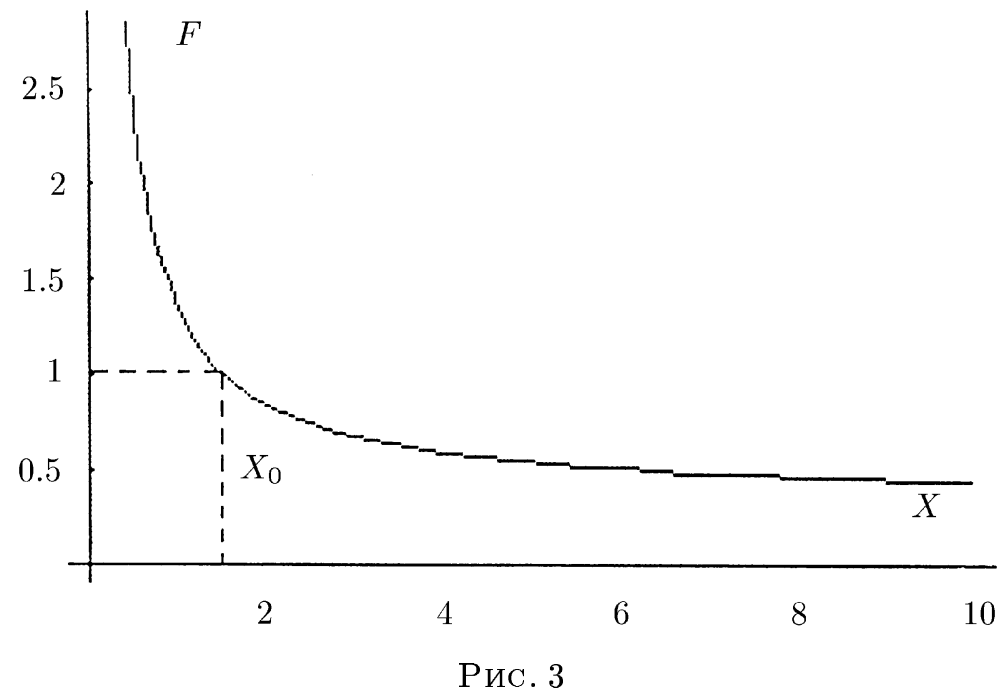

ЗАмЕчАниЕ 2.1. Общее решение дифференциального уравнения модулящий (2.15) является решением следующего уравнения:

$$
\int_{F_{0}}^{F(X)} \frac{d f}{\frac{17}{16}\left(f+\sqrt{f^{2}-1}\right) \frac{E}{K}-2 f}=\log X .
$$

Типичное поведение параметра $F$ как функции от $X$ отражено на рис. 3 .

ЗАмЕЧАниЕ 2.2. Условие (2.14) определяет эллиптический анзатц (2.4) следующим образом (функции Вейерштрасса выбраны исходя из соображений простоты их интегрирования):

$$
u_{0}=\pi(2 n+1)-i \log \left\{4\left[\wp\left(x+\varphi \mid g_{2}, g_{3}\right)\right]\right\}, \quad n \in \mathbb{Z}
$$

где $\wp-ф у$ фнкия Вейерштрасса, $g_{2}, g_{3}$ - задающие ее параметры;

$$
g_{2}=\frac{1}{3} F^{2}-\frac{1}{4}, \quad g_{3}=\frac{1}{24} F-\frac{1}{27} F^{3} ; \quad e_{1}=\frac{1}{6} F, \quad e_{2,3}=-\frac{1}{12} F \pm \frac{1}{4} \sqrt{F^{2}-1} .
$$

Формула (2.17) может быть переписана в терминах функций Якоби, что иллюстрирует соответствие (2.17) решению уравнения математического маятника. Эллиптическая функция в формуле (2.17) претерпевает вырождение в случае $x=0(F \rightarrow \infty$, модуль соответствующего синуса Якоби обращается в нуль) и в случае конечного значения переменной $\varepsilon x_{0}=X_{0}$, где $F\left(X_{0}\right)=1$ и модуль синуса Якоби равен 1 . Последнее вырождение может быть описано на язьке функций Вейерштрасса как слияние вещественных точек ветвления $e_{2,3}$ и превращение их в комплексно-сопряженную пару.

Последующая часть данной работы посвящена асимптотической по малому параметру $\varepsilon=\alpha^{-1}, \alpha \rightarrow \infty$ (см. (1.2)) оценке сдвига фазы $\varphi$ в формуле (2.17).

3. Линейная задача и метод изомонодромных деформаций. Рассмотрим линейное матричное дифференциальное уравнение

$$
D_{\lambda} \Psi=A \Psi
$$


где $A$ задана формулой $(2.11), \Psi$ - неизвестная $2 \times 2$-матрица. Проведем замену переменных $x=\alpha X, \xi=\alpha \lambda$ и с учетом (2.14) получим из (3.1) следующее уравнение:

$$
\begin{gathered}
D_{\xi} \Psi=A \Psi \\
A=\alpha\left[i \sigma_{3}\left(-\frac{X^{2}}{16}+\frac{\cos u}{\xi^{2}}\right)-\frac{i X \sqrt{2 F+2 \cos u}}{4 \xi} \sigma_{1}+\frac{i \sin u}{\xi^{2}} \sigma_{2}\right] .
\end{gathered}
$$

Пусть $\mu(\xi)$ - собственные значения матрицы $A$, точки поворота $\xi_{j}, j=1,2,3,4,-$ нули функции $\mu(\xi)$. В плоскости комплексных значений параметра $\xi$ условиями

$$
\operatorname{Re}\left[i \alpha \int_{\xi_{j}}^{\xi} \mu(\zeta) d \zeta\right]=0
$$

определим так назьваемые линии Стокса (см. рис. 4). Они делят плоскость на семь областей $\Omega_{k}, k=0,1,2, \ldots, 6$. В каждой из них определим каноническое решение задачи (3.2), удовлетворяющее условию нормировки:

$$
\begin{gathered}
\Psi_{j}=\left[I+O\left(\xi^{-1}\right)\right] \exp \left(-\frac{i \alpha X^{2}}{16} \xi \sigma_{3}\right), \quad \xi \rightarrow \infty, \quad \xi \in \Omega_{j}, \quad j \neq 0 \\
\Phi=\Psi_{0}=i\left[I+O\left(\xi^{-1}\right)\right] \exp \left(-i \alpha \xi^{-1} \sigma_{3}\right), \quad \xi \rightarrow 0, \quad \xi \in \Omega_{0}
\end{gathered}
$$
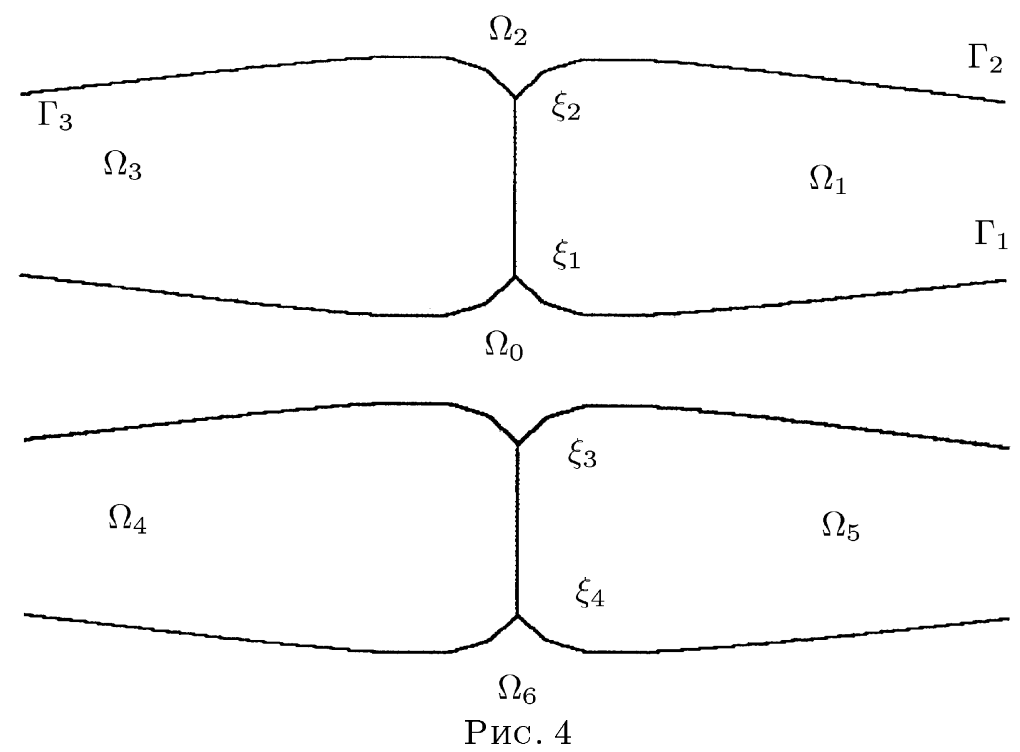

Следующим образом зададим матрицы Стокса:

$$
\Psi_{j+1}=\Psi_{j} S_{j}, \quad j=0,1, \ldots, 6
$$


Теорема 3.1 (ср. с [9], [7]). Функиия и, определяющая формулу (3.2), удовлетворяет уравнению $P_{3}(2.10)$ тогда и только тогда, когда матрицы Стокса $S_{j}$ не зависят от переменной $x$.

Теперь рассмотрим задачу (3.2) в точках $X_{n}$ таких, что $\cos u\left(X_{n}\right)=1$ :

$$
D_{\xi} \Psi=-i \alpha\left[\sigma_{3}\left(\frac{X^{2}}{16}-\xi^{-2}\right)+\frac{X \sqrt{2 F+2}}{4 \xi} \sigma_{1}\right]
$$

Здесь и далее для краткости вместо $X_{n}$ пишем $X$.

Очевидно, что, вычислив величины $X_{n}$, мы сможем легко оценить сдвиг фазы $\varphi$ эллиптической функции (2.17). Следующий пункт будет посвящен приближенному вычислению матриц Стокса. Для этого понадобится использование аппарата метода ВКБ и теории спецфункций.

4. Построение приближенной $\Psi$-функции вдали от точек поворота и в их малой окрестности. Система (3.6) имеет большой параметр $\alpha$ в правой части, поэтому ее решения допускают ВКБ-приближения следующего вида:

$$
\Psi=\left[T+O\left(\alpha^{-1} \mu^{-3 / 2}\right)\right] \exp \left[-i \alpha \int_{\xi_{0}}^{\xi}\left(\mu \sigma_{3}-T^{-1} D_{\zeta} T\right) d \zeta\right] C
$$

где

$$
\begin{gathered}
T=\sqrt{\frac{d_{1}}{\mu}}\left(\begin{array}{cc}
1 & \frac{d_{1}-\mu}{d_{2}} \\
\frac{\mu-d_{1}}{d_{2}} & 1
\end{array}\right), \quad d_{1}=\frac{X^{2}}{16}-\xi^{-2}, \quad d_{2}=\frac{X \sqrt{2 F+2}}{4 \xi} \\
\mu=\frac{X \sigma_{3}}{16 \xi^{2}} \sqrt{\xi^{4}+\frac{32 F}{X^{2}} \xi^{2}+\frac{256}{X^{4}}}
\end{gathered}
$$

Точки поворота $\xi_{j}, j=1,2,3,4,-$ нули функции $\mu(\xi)$ :

$$
\xi_{1,2,3,4}=\frac{4 i}{X}\left[(-1)^{n} \sqrt{F+1}+(-1)^{m} \sqrt{F-1}\right] ; \quad m, n=1,2
$$

$C$ - некоторая постоянная по $\xi$ матрица.

Уравнение (4.2) задает риманову поверхность $(\mu, \xi)$, канонические циклы на которой определим так, как показано на рис. 5. Тогда вьполняется следующая лемма.

ЛЕмма 4.1. Фазовые интегралы в формуле (4.1) удовлетворяют следуюшим ои,енкам:

$$
\begin{aligned}
& \int_{\xi_{2}}^{\xi} \mu(\zeta) d \zeta=\frac{X^{2}}{16} \xi-\frac{1}{4} \oint_{2 b-a} \mu(\zeta) d \zeta+O\left(\xi^{-1}\right), \quad \xi \in \Gamma_{2}, \quad \xi \rightarrow \infty \\
& \int_{\xi_{1}}^{\xi} \mu(\zeta) d \zeta=\frac{X^{2}}{16} \xi+\frac{1}{4} \oint_{a} \mu(\zeta) d \zeta+O\left(\xi^{-1}\right), \quad \xi \in \Gamma_{1}, \quad \xi \rightarrow \infty
\end{aligned}
$$




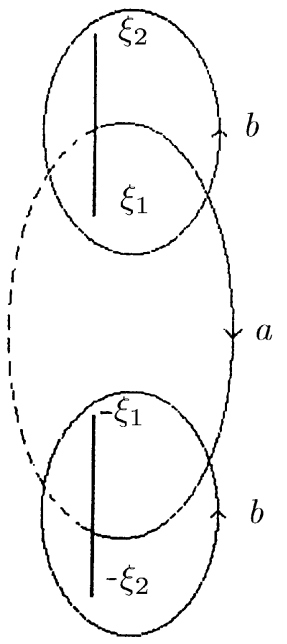

Рис. 5

ДокАЗАТЕЛЬСТво использует следующее наблюдение:

$$
\int_{\Gamma_{2} \cup \Gamma_{3}} \mu(\zeta) d \zeta=\left[\int_{\xi_{2}}^{-\xi_{2}}-\oint_{b-a / 2}\right] \mu(\zeta) d \zeta
$$

Формула (4.5) доказьвается аналогичным образом.

Определим матричные функции $\Psi_{j}$ в областях $\Omega_{j}, j=1,2,3$, как решения системы (3.6) с граничным условием

$$
\Psi_{j}=\left[I+O\left(\xi^{-1}\right)\right] \exp \left(-\frac{i \alpha X^{2} \xi}{16} \sigma_{3}\right), \quad \xi \in \Gamma_{j}, \quad \xi \rightarrow \infty .
$$

ТЕОРема 4.1. Канонические решения $\Psi_{j}$ системы (3.6), нормированные граничным условием (4.5), имеют следуюшую асимптотику при $\alpha \rightarrow \infty$ :

$$
\begin{aligned}
& \Psi_{1}(\xi)=T[1+o(1)] \exp \left[-i \alpha \sigma_{3}\left(\int_{\xi_{1}}^{\xi} \mu(\zeta) d \zeta-\frac{1}{4} \oint_{a} \mu(\zeta) d \zeta\right)\right], \quad \xi \in \Omega_{1} \\
& \Psi_{3}(\xi)=T[1+o(1)] \exp \left[-i \alpha \sigma_{3}\left(\int_{\xi_{2}}^{\xi} \mu(\zeta) d \zeta+\frac{1}{4} \oint_{2 b-a} \mu(\zeta) d \zeta\right)\right], \quad \xi \in \Omega_{3} .
\end{aligned}
$$

ДоКАЗАТЕЛЬСТво. Формулы (4.7) и (4.8) отличаются от (4.1) лишш выбором правого матричного множителя $C$. По лемме 4.1 функции $\Psi_{1}, \Psi_{3}$ имеют асимптотику (4.6) на кривых $\Gamma_{1}, \Gamma_{3}$ соответственно. Внутри областей $\Omega_{1}, \Omega_{3}$ функции $\Psi_{1}, \Psi_{3}$ приближенно удовлетворяют уравнению (3.6) в соответствии с теорией ВКБ.

Легко видеть, что ВКБ-приближение (4.7), (4.8) справедливы лишь вне достаточно малой окрестности точек поворота $\xi_{j}$. Однако, матрицы Стокса станем вычислять путем продолжения функций $\Psi_{1}, \Psi_{3}$ на отрезок $\left[\xi_{1}, \xi_{2}\right]$, что легче сделать с помощью анализа их поведения именно в окрестности точек поворота. 
Лемма 4.2. Существуют решения системы (3.6) в окрестности точек поворота $\xi_{j}, j=1,2$, имеющие асимптотику

$$
\Psi(\xi, \alpha, X)=\alpha^{1 / 6}\left(\begin{array}{cc}
\omega(\zeta) & -i \bar{\omega}(\zeta) \\
i \omega(\zeta) & \bar{\omega}(\zeta)
\end{array}\right) D+O\left[\alpha^{-1 / 6} \zeta^{3 / 4} \exp \left( \pm 4 i \gamma_{j} \zeta^{3 / 2}\right)\right]
$$

әде $\xi \in \Gamma_{j}, \zeta=\alpha^{2 / 3}\left(\xi-\xi_{j}\right), \gamma_{j}^{2}=X^{4}\left(\xi_{j}^{2}-\xi_{3-j}^{2}\right) /\left(4608 \xi_{j}^{3}\right), j=1,2, D$ - постоянная по $\zeta$ матрича, а функиии $\omega, \bar{\omega}$ - линейно независимые решения уравнения Эйри

$$
\omega_{\zeta \zeta}+36 \gamma^{2} \zeta \omega=0
$$

ДокАЗАТЕЛЬСТВо. Легко убедиться, что в окрестности точки $\xi_{j}$ фазовая функция $\mu$ имеет асимптотику

$$
\mu=6 \gamma_{j} \alpha^{-1 / 3} \zeta^{1 / 2}+O\left(\left(\xi-\xi_{j}\right)^{3 / 2}\right) .
$$

Пусть $\psi_{1}, \psi_{2}$ - компоненты вектор-столбца матрицы $\Psi$. Тогда задача (3.6) эквивалентна следующей системе:

$$
\chi_{1, \xi}=-\frac{i \alpha X^{2}}{16 \xi^{2}}\left(\xi-\xi_{1}\right)\left(\xi-\xi_{2}\right) \chi_{2}, \quad \chi_{2, \xi}=-\frac{i \alpha X^{2}}{16 \xi^{2}}\left(\xi+\xi_{1}\right)\left(\xi+\xi_{2}\right) \chi_{1},
$$

где $\chi_{1,2}=\psi_{1} \pm i \psi_{2}$. Из (4.12) выводим уравнение

$$
\chi_{2, \xi \xi}=\frac{i \alpha X^{2}}{16 \xi^{2}}\left(\xi_{1}+\xi_{2}+2 \frac{\xi_{1} \xi_{2}}{\xi}\right) \chi_{1}-\frac{\alpha^{2} X^{4}}{256 \xi^{4}}\left(\xi^{2}-\xi_{1}^{2}\right)\left(\xi^{2}-\xi_{2}^{2}\right) \chi_{2} .
$$

В окрестности точки поворота $\xi_{j}$ ищем решения уравнения (4.13) в виде ряда с главными членами $\omega_{1,2}$ :

$$
\chi_{1,2}=\omega_{1,2}+O\left(\alpha^{-1 / 3}\right)
$$

Подставляя (4.14) в (4.13), получим в главном порядке

$$
\omega_{2, \zeta \zeta}+36 \gamma_{j}^{2} \zeta \omega_{2}=0, \quad \omega_{1}=0
$$

где $\zeta=\alpha^{2 / 3}\left(\xi-\xi_{j}\right)$. Отсюда вьводим оценки

$$
\psi_{1}=\frac{1}{2} \omega_{2}+O\left(\alpha^{-1 / 3}\right), \quad \psi_{2}=\frac{i}{2} \omega_{2}+O\left(\alpha^{-1 / 3}\right)
$$

и вместе с ними требование леммы.

Асимптотическое поведение функций Эйри на линиях Стокса известно (см. [6]), поэтому мы можем фиксировать базисные решения $\omega, \bar{\omega}$ уравнения Эйри (4.10) асимптотиками на лучах $\arg \zeta=-\frac{2}{3} \arg \gamma$ :

$$
\begin{aligned}
& \omega(\zeta)=\zeta^{-1 / 4}\left[1+O\left(\zeta^{-3 / 2}\right)\right] \exp \left(-4 i \gamma \zeta^{3 / 2}-\frac{\pi i}{4}\right), \quad \zeta \rightarrow \infty \\
& \bar{\omega}(\zeta)=\zeta^{-1 / 4}\left[1+O\left(\zeta^{-3 / 2}\right)\right] \exp \left(4 i \gamma \zeta^{3 / 2}+\frac{\pi i}{4}\right),
\end{aligned}
$$

Тогда эти функции удовлетворяют следующим условиям:

$$
\begin{aligned}
\bar{\omega}\left(\zeta e^{-2 \pi i / 3}\right) & =e^{-\pi i / 3}[\bar{\omega}(\zeta)-\omega(\zeta)], & \bar{\omega}\left(\zeta e^{2 \pi i / 3}\right) & =e^{\pi i / 3} \omega(\zeta), \\
\omega\left(\zeta e^{2 \pi i / 3}\right) & =e^{\pi i / 3}[\omega(\zeta)-\bar{\omega}(\zeta)], & \omega\left(\zeta e^{-2 \pi i / 3}\right) & =e^{-\pi i / 3} \bar{\omega}(\zeta)
\end{aligned}
$$

С помощью этих соотношений продолжим функции $\Psi_{1}$ и $\Psi_{3}$ на отрезок $\left[\xi_{1}, \xi_{2}\right]$. 
Теорема 4.2. Канонические решения $\Psi_{j}, j=1,2,3$, имеют следующие асимптотики на отрезке $\left[\xi_{1}, \xi_{2}\right]$ :

$$
\begin{gathered}
\Psi_{1,3}(\xi)=\sqrt{6} T\left[1+O\left(\alpha^{-1} \mu^{-3 / 2}\right)\right] \exp \left[-i \alpha \sigma_{3} \int_{\xi_{1,2}}^{\xi} \mu(\zeta) d \zeta\right]\left(\begin{array}{ll}
1 & 1 \\
0 & 1
\end{array}\right) D_{j}, \quad j=1,2 \\
\Psi_{2}(\xi)=\sqrt{6} T\left[1+O\left(\alpha^{-1} \mu^{-3 / 2}\right)\right] \exp \left[-i \alpha \sigma_{3} \int_{\xi_{2}}^{\xi} \mu(\zeta) d \zeta\right]\left(\begin{array}{ll}
1 & 0 \\
1 & 1
\end{array}\right) D_{2}, \\
D_{1}=\exp \left[\frac{i \alpha}{4} \oint_{a} \mu(\zeta) d \zeta\right], \quad D_{2}=\exp \left[\frac{i \alpha}{4} \oint_{2 b-a} \mu(\zeta) d \zeta\right] .
\end{gathered}
$$

ДокАЗАТЕЛЬСТво. Для вывода формулы (4.18) рассмотрим поведение $\Psi$-функции в окрестности точки $\xi_{1}$. Формулы (4.7) и (4.9) описывают решения одной системы (3.6), поэтому в некоторой общей области, где переменная $\xi-\xi_{1}$ мала, а $\zeta$ велика, они должны отличаться на постоянньй множитель. Этот множитель $D$ в формуле (4.9) определим из условия на асимптотики на границе $\Gamma_{1}$ в окрестности точки $\xi_{1}$. Оценим величину (4.7) при $\xi \rightarrow \xi_{1}, \xi \in \Gamma_{1}$ :

$$
\begin{aligned}
\Psi_{1}= & {\left[\alpha^{1 / 6} \zeta^{-1 / 4} \sqrt{\frac{d_{1}}{6 \gamma_{1}}}\left(\begin{array}{cc}
1 & -i \\
i & 1
\end{array}\right)+O\left(\alpha^{-1 / 2} \zeta^{-3 / 4}\right)\right] } \\
& \times \exp \left\{-i \sigma_{3}\left[4 \gamma_{1} \zeta^{3 / 2}+O\left(\alpha^{-2 / 3} \zeta^{5 / 2}\right)\right]\right\} D_{1} .
\end{aligned}
$$

Подставив асимптотики функций Эйри (4.16) в формулу (4.9) и сравнив главные члены с формулой (4.21), получим

$$
D=\sqrt{\frac{d_{1}}{\gamma_{1}}} \exp \left(\frac{\pi i \sigma_{3}}{4}\right) D_{1}
$$

Для продолжения функции $\Gamma_{1}$ на отрезок $\left[\xi_{1}, \xi_{2}\right]$ нужно в формуле (4.9) $\zeta$ заменить на $\zeta e^{2 \pi i / 3}$. С помощью (4.17) получаем

$$
\begin{aligned}
\underset{\xi \in\left[\xi_{1}, \xi_{2}\right]}{\Psi_{1}(\xi, \alpha)}=\left.\Psi_{1}\left(\xi_{1}+\zeta \alpha^{-2 / 3} e^{-2 \pi i / 3}, \alpha\right)\right|_{\zeta e^{2 \pi i / 3}} \\
=\alpha^{1 / 6}\left(\begin{array}{cc}
\omega\left(\zeta e^{-2 \pi i / 3}\right) & -i \bar{\omega}\left(\zeta e^{-2 \pi i / 3}\right) \\
i \omega\left(\zeta e^{-2 \pi i / 3}\right) & \bar{\omega}\left(\zeta e^{-2 \pi i / 3}\right)
\end{array}\right)_{\zeta e^{2 \pi i / 3}} D+O\left(\alpha^{-1 / 6} \zeta^{3 / 4} \exp \left( \pm 4 i \gamma_{1} \zeta^{3 / 2}\right)\right) \\
=\alpha^{1 / 6} e^{-\pi i / 3}\left(\begin{array}{cc}
\bar{\omega}(\zeta) & -i[\bar{\omega}(\zeta)-\omega(\zeta)] \\
i \omega(\zeta) & \bar{\omega}(\zeta)-\omega(\zeta)
\end{array}\right)_{\zeta e^{2 \pi i / 3}} D+O\left(\alpha^{-1 / 6} \zeta^{3 / 4} \exp \left( \pm 4 i \gamma_{1} \zeta^{3 / 2}\right)\right) \\
=\alpha^{1 / 6} e^{-\pi i / 3}\left(\begin{array}{cc}
\bar{\omega} & -i \omega \\
i \bar{\omega} & \omega
\end{array}\right)_{\zeta e^{2 \pi i / 3}}\left(\begin{array}{cc}
1 & i \\
0 & -1
\end{array}\right) D+O\left(\alpha^{-1 / 6} \zeta^{3 / 4} \exp \left( \pm 4 i \gamma_{1} \zeta^{3 / 2}\right)\right) \\
=-i \alpha^{1 / 6} \zeta^{-1 / 4}\left(\begin{array}{cc}
1 & -i \\
i & 1
\end{array}\right) \exp \left[i \sigma_{3}\left(4 \gamma_{1} \zeta^{3 / 2} e^{\pi i}+\frac{\pi}{4}\right)\right]\left(\begin{array}{cc}
1 & -i \\
0 & -1
\end{array}\right) D \\
\quad+O\left(\alpha^{-1 / 6} \zeta^{3 / 4} \exp \left( \pm 4 i \gamma_{1} \zeta^{3 / 2}\right)\right) .
\end{aligned}
$$


С другой стороны, функция $\Psi_{1}$ имеет на отрезке $\left[\xi_{1}, \xi_{2}\right]$ вид $(4.1)$ с некоторьм правьп множителем $C_{1}$. Сравнивая асимптотику (4.1) на отрезке $\left[\xi_{1}, \xi_{2}\right]$ при $\xi \rightarrow \xi_{1}$ и $(4.23)$ при $\zeta \rightarrow \infty$, вьводим

$$
C_{1}=\sqrt{6}\left(\begin{array}{ll}
1 & 1 \\
0 & 1
\end{array}\right) D_{1}
$$

откуда вытекает формула (4.18). Аналогичным образом могут быть вьведены формулы (4.19) и (4.20), причем в последнем случае поворот по переменной $\zeta$ в (4.9) в окрестности точки $\xi_{2}$ производится против часовой стрелки.

5. Асимптотическое распределение нулей и сдвиг фазы эллиптического анзатца. Представления для $\Psi$-функций (4.18)-(4.20) позволяют явно оценить матрицы Стокса $S_{1}$ и $S_{2}$ (см. (3.5)) как выражения через параметры $F, X, \alpha$, определяющие коэффищиенты системы (3.6). Процедура обращения таких формул дает асимптотическое описание распределения нулей $X_{m}$ функции $\sin u$

Величины $S_{1}^{-1}=\Psi_{2}^{-1} \Psi_{1}$ и $\left(S_{2} S_{1}\right)^{-1}=\Psi_{3}^{-1} \Psi_{1}$ станем вычислять на отрезке $\left[\xi_{1}, \xi_{2}\right]$ (см. рис. 2). Для этого используем формулы (4.18)-(4.20).

ТЕОрема 5.1. Матрицы Стокса могут быть представлены следуюшими формулами:

$$
S_{1}=\left(\begin{array}{cc}
1+\frac{1}{g} & -\frac{1}{h} \\
\frac{h}{g} & 1
\end{array}\right), \quad S_{2} S_{1}=\left(\begin{array}{cc}
1 & \frac{g-1}{h} \\
0 & 1
\end{array}\right)
$$

$2 \partial e$

$$
\begin{gathered}
h=\exp \left[\frac{i \alpha}{2} \oint_{a} \mu(\zeta) d \zeta\right], \quad g=\exp \left[i \alpha \oint_{b} \mu(\zeta) d \zeta\right] \\
\int \mu(\zeta) d \zeta=-\frac{X_{m}}{4} \int \frac{1}{\nu^{2}} \sqrt{\nu^{4}+F \nu^{2}+1} d \nu .
\end{gathered}
$$

ДоКАЗАТЕЛЬСТво. С помощью формул (4.18), (4.19) получаем следующее:

$$
\Psi_{3}^{-1} \Psi_{1}=D_{2}^{-1}\left(\begin{array}{cc}
1 & -1 \\
0 & 1
\end{array}\right) \exp \left[i \alpha \sigma_{3} \int_{\xi_{2}}^{\xi_{1}} \mu(\zeta) d \zeta\right]\left(\begin{array}{ll}
1 & 1 \\
0 & 1
\end{array}\right) D_{1}=\left(\begin{array}{cc}
1 & \frac{1-g}{h} \\
0 & 1
\end{array}\right) .
$$

Аналогичным образом выводится соотношение

$$
\Psi_{2}^{-1} \Psi_{1}=\left(\begin{array}{cc}
1 & \frac{1}{h} \\
\frac{h}{g} & 1+\frac{1}{g}
\end{array}\right)
$$

что дает требуемое.

Теперь, считая $g, h$ фиксированными параметрами, разрешим равенства (5.1) относительно величины $X_{m}$. Легко выразить интегралы по циклам $a, b$ фазовой функции $\mu(\nu)$ через полные эллиптические интегралы

$$
\oint \mu(\zeta) d \zeta=-\frac{i X}{4 \sqrt{2 F+2}} \oint \sqrt{1-k^{2} \sin ^{2} t} d t
$$


откуда следует

$$
\oint_{a} \mu(\zeta) d \zeta=-i X \sqrt{\frac{F+1}{8}} E, \quad \oint_{b} \mu(\zeta) d \zeta=-i X \sqrt{\frac{F+1}{8}} E^{\prime}, \quad k^{2}=\frac{2}{F+1} .
$$

Периоды соответствующей эллиптической функции определяются соотношением

$$
\omega=k K, \quad \omega^{\prime}=i k K^{\prime} .
$$

Станем искать нули $X_{m}$ в виде асимптотических рядов по малому параметру $\alpha^{-1}$ :

$$
\alpha X_{m}=2 m \omega+z_{0}+O\left(\alpha^{-1}\right) ;
$$

из соображений вещественности аргумента в формулу (5.4) входит только вещественный период $2 \omega$. Следовательно, имеем

$$
i \alpha \oint_{a, b} \mu(\zeta) d \zeta=-\alpha X_{m} \sqrt{\frac{F+1}{8}} E^{(\prime)}=-m K E^{(\prime)}-z_{0} E^{(\prime)} \sqrt{\frac{F+1}{8}} .
$$

Используя (5.1), вьводим следующую систему:

$$
\begin{aligned}
2 \log h & =-i \alpha \oint_{a} \mu(\zeta) d \zeta=-m K E-\frac{z_{0} E}{2 k} \\
\log g & =-i \alpha \oint_{b} \mu(\zeta) d \zeta=-m K E^{\prime}-\frac{z_{0} E^{\prime}}{2 k}
\end{aligned}
$$

откуда с помощью тождества Лежандра $E K^{\prime}+E^{\prime} K-K K^{\prime}=\pi / 2$ получаем равенства по модулю $\pi m$ :

$$
2 \log h=-m K E-\frac{z_{0} E}{2 k}, \quad 2 \log h=-2 m K\left(K^{\prime}-E^{\prime}\right)-\frac{2 z_{0} E^{\prime}}{k} .
$$

Решая последнюю систему относительно $z_{0}$, выводим

$$
z_{0}=\frac{2 k}{E\left(2 E^{\prime}-K^{\prime}\right)}\left[2\left(K^{\prime}-E^{\prime}\right) \log h-E \log g\right], \quad k^{2}=\frac{2}{F+1} .
$$

Результатом этих вычислений является следующая теорема

Теорема 5.2. Радиально-симметричное сингулярное в нуле решение уравнения синус-Гордон (1.1) для случая $f=0$, описываемое третьим трансиендентом Пенлеве (2.10), с особенностью вида

$$
u=\alpha \log \frac{1}{x}+\beta+o(1), \quad x \rightarrow 0,
$$

мохет быть представлено в виде асимптотического по малому параметру $\alpha^{-1}$, $\alpha \rightarrow \infty$, ряда (2.5) с главнымии членами

$$
u=\alpha \log x+\pi-i \log \left\{4\left[\wp\left(x+z_{0} \mid g_{2}, g_{3}\right)-\frac{1}{6} F\right]\right\}+o(1),
$$

$2 \partial e$

$$
g_{2}=\frac{1}{3} F^{2}-\frac{1}{4}, \quad g_{3}=\frac{1}{24} F-\frac{1}{27} F^{3},
$$

$\wp-p$-функиия Вейеритрасса, величина $F$ удовлетворяет уравнению модулячий (2.15), а сдвиг фазы $z_{0}$ определен формулой (5.5). 


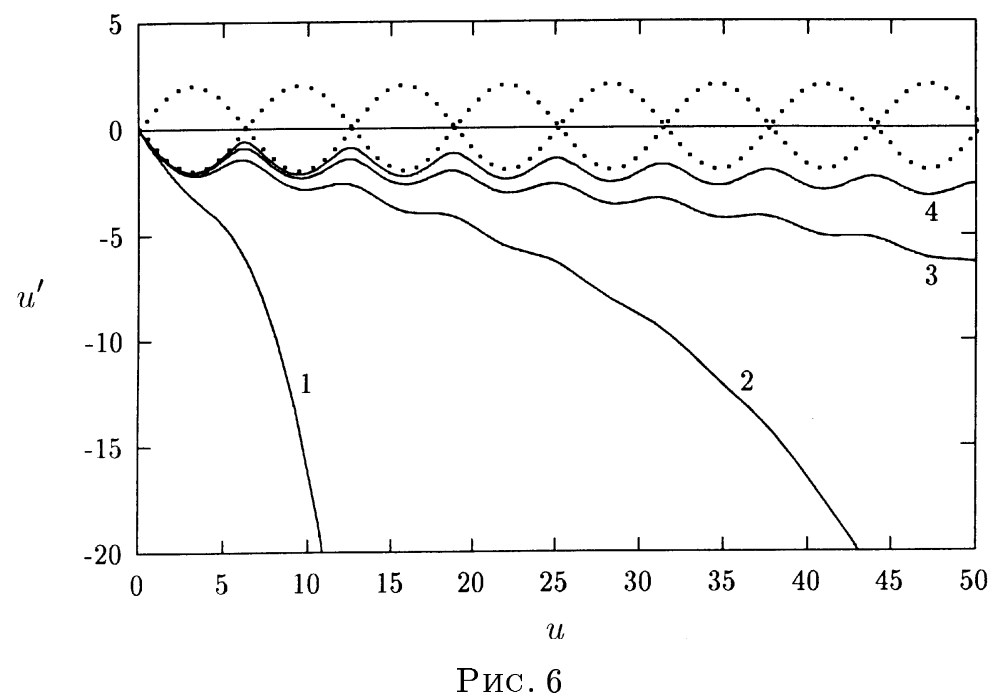

ДоКАЗАТЕЛЬСТво заключается в проверке вьполнения условия (2.9) для $j=3$ и формулы (5.6). Члены порядка $O\left(\varepsilon^{-1}\right)$ исчезают очевидным образом. Выполнение равенства в порядке $O(1)$ обеспечивает метод Уизема, строящий член асимптотического ряда $u_{0}(x)$ в виде возмущения решения уравнения математического маятника (2.14). Последний факт наглядно иллюстрируется полученной численньм путем в работе [1] фазовой картиной для решения $P_{3}(2.10)-(1.2)$ (см. рис. 6).

Кривые пронумерованы в порядке возрастания значения параметра $\alpha$.

В заключение автор выражает глубокую признательность В. Ю. Новокшенову за постановку задачи и постоянную помощь.

\section{СПИСОК ЦИТИРОВАННОЙ ЛИТЕРАТУРЫ}

[1] Новокшенов В. Ю., Шагалов А. Г. Решения типа связанных состояний для эллиптического уравнения синус-Гордон // ТМФ. 1997. Т. 111. № 1. С. 15-31.

[2] Новокшенов В. Ю. Об асимптотике общего вещественного решения третьего уравнения Пенлеве // Докл. АН СССР. 1985. Т. 283. №5. С. 1161-1165.

[3] Novokshenov V.Yu. Radial-symmetric solution of the cosh-Laplace equation and the distribution of its singularities. Preprint № 64, SFB 288. Berlin, 1993.

[4] Vereschagin V. L. Nonlinear quasiclassics and the Painlevé equations // solv-int/9707004. 1997.

[5] Итс А. Р. "Изомонодромные" решения уравнений нулевой кривизны // Изв. АН СССР. Сер. матем. 1985. Т. 49. №3. С. 32-63.

[6] Федорюк М.В. Асимптотические методы для линейных обыкновенных дифференциальных уравнений. М.: Наука, 1983.

[7] Jimbo M., Miwa T. Monodromy preserving deformation of linear ordinary differential equations with rational coefficients. II // Phys. D. 1981. V. 2. №3. P. 407-448.

[8] Бейтмен Г., Ердейи А. Высшие трансцендентные функции. Т. 3. М.: Наука, 1967.

[9] Flaschka H., Newell A. C. Monodromy and spectrum preserving deformations. I / Comm. Math. Phys. 1980. V. 76. P. 67-116. 